\title{
Nanoscale Analysis by EFTEM and FIB-Tomography for Optimization of Thin-Film Silicon Solar Cells
}

\author{
D.T.L. Alexander, ${ }^{*}$ S. Nicolay,** P. Cuony,** M. Cantoni,** and C. Ballif** \\ * Centre Interdisciplinaire de Microscopie Électronique (CIME), École Polytechnique Fédérale de \\ Lausanne (EPFL), Lausanne, CH-1015, Switzerland \\ ** Institute of Microengineering (IMT), École Polytechnique Fédérale de Lausanne (EPFL), \\ Neuchâtel, CH-2000, Switzerland
}

To produce high-efficiency thin-film Si micromorph (tandem of amorphous and microcrystalline junction) cells requires optimization of all thin-film components. To this aim, we high-light the contribution of advanced electron microscopy to the optimization of plasma-enhanced chemical vapor deposition (PE-CVD) $\mathrm{SiO}_{\mathrm{x}}$ and low-pressure (LP-) CVD ZnO layers for micromorph cells.

The PE-CVD $\mathrm{SiO}_{\mathrm{x}}$ films are used as intermediate reflectors, combining desireable properties of a low refractive index for minimal light absorption, with a high transplanar, but low in-plane, conductivity to channel current between the two junctions [1]. Although known to be a composite of conductive $\mathrm{Si}$ nanoparticles ( $\mathrm{Si} \mathrm{NPs}$ ) in an insulating $\mathrm{SiO}_{2}$ matrix, the microstructure giving their particular conductivity properties was previously unknown. Now, cross-section energy-filtered (EF-) TEM imaging of test films finds that the Si NPs grow in branched/dendritic networks that connect the two surfaces of the films, producing transplanar conductive routes, as shown in Fig. 1a. The image is taken with an $\Omega$-filter on a JEOL 2200FS, using a $4 \mathrm{eV}$ window centred on the $17.5 \mathrm{eV} \mathrm{Si}$ plasmon peak. NP definition is improved by removing the intensity contribution from the broad $\mathrm{SiO}_{2}$ plasmon peak. This is done by adapting the STEM-EELS approach of Schamm et al. [2] to EFTEM, using a linear fit between 10 and $23 \mathrm{eV}$ filtered images to model, and then subtract, the $\mathrm{SiO}_{2}$ intensity contribution. Extension of the analysis to more cell-like conditions of growth on pyramidal $\mathrm{ZnO}$ shows that the conductive transplanar networks are maintained, but that their formation and connectivity clearly depends on the underlying $\mathrm{ZnO}$ morphology [Fig. 1b].

The pyramidal $\mathrm{ZnO}$ structures shown in Fig. 1b are used as transparent conductive oxide layers, and play an essential role in scattering light into the junctions to increase cell efficiency. In order to optimize the growth of such surface architectures, their formation, which begins with competitive growth of randomly-oriented nanocrystalline nuclei on an amorphous glass substrate, must be understood [3]. Being non-epitaxial, the films have complex grain structures that are challenging to analyse. However, we find that FIB-SEM cross-section imaging of the films with a Zeiss NVision 40 provides an excellent tool for their analysis. By using a $1.5 \mathrm{kV}$ electron beam, and an in-lens backscattered-electron detector, grains $<20 \mathrm{~nm}$ in diameter can be distinguished with a contrast superior to BF or DF TEM imaging (Fig. 2a). Slice-and-view tomography extends this approach to the third dimension. Fig. $2 \mathrm{~b}$ shows a small portion of data from such an acquisition for a $\mathrm{ZnO}$ test film, with a basic voxel size of $10 \times 10 \times 10 \mathrm{~nm}$. The transition from high nucleation density to grain grain coarsening, driven by competitive growth, is clear. Such a data set provides extensive scope for analysis. For instance, by correlating with XRD data, we know that the pyramids of this film are oriented parallel to [110]. Now, the FIB data shows that they grow with 2-fold rotational symmetry around axial twin planes (Fig. 2b). TEM studies are underway to investigate the growth axes of such $\mathrm{ZnO}$ films more deeply, along with identifying the crystallinity of the $\mathrm{Si} \mathrm{NPs}_{\text {in }}$ the $\mathrm{SiO}_{\mathrm{x}}$ films. 
References

[1] Buehlmann et al., Appl. Phys. Lett., 91 (2007) 143505.

[2] Schamm et al., Ultramicroscopy, 108 (2008) 346.

[3] Nicolay et al., Cryst. Growth Design, 9 (2009) 4957.

[4] The Swiss Federal Office for Energy (OFEN) is acknowledged for financial support.

(a)

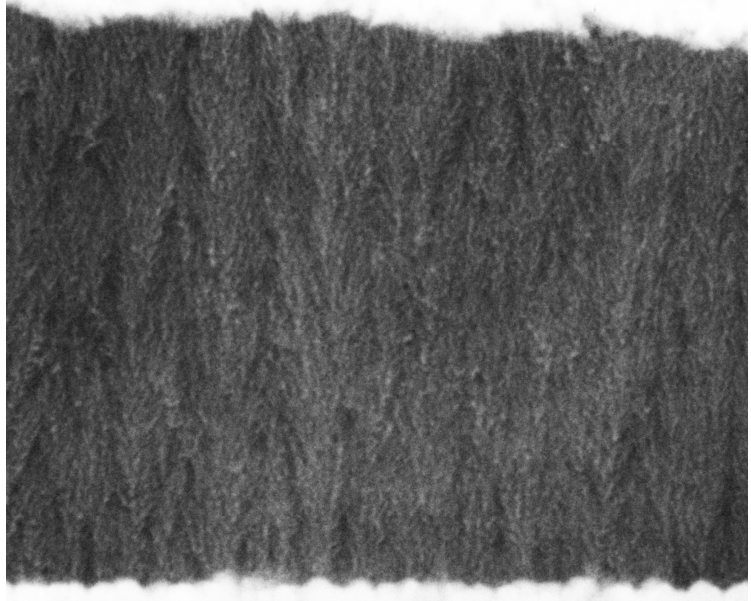

$50 \mathrm{~nm}$

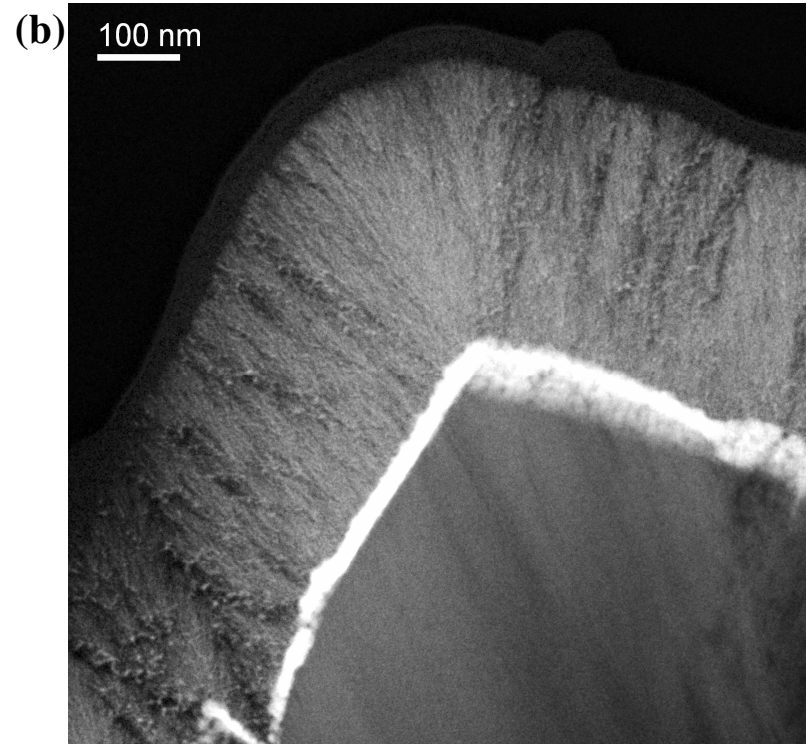

FIG. 1. Cross-section EFTEM images of Si NPs in $\mathrm{SiO}_{\mathrm{x}}$ films made on flat Si wafer (a) and on pyramidal $\mathrm{ZnO}$ (b). Branched/dendritic conductive pathways of Si NPs cross the films.

(a)

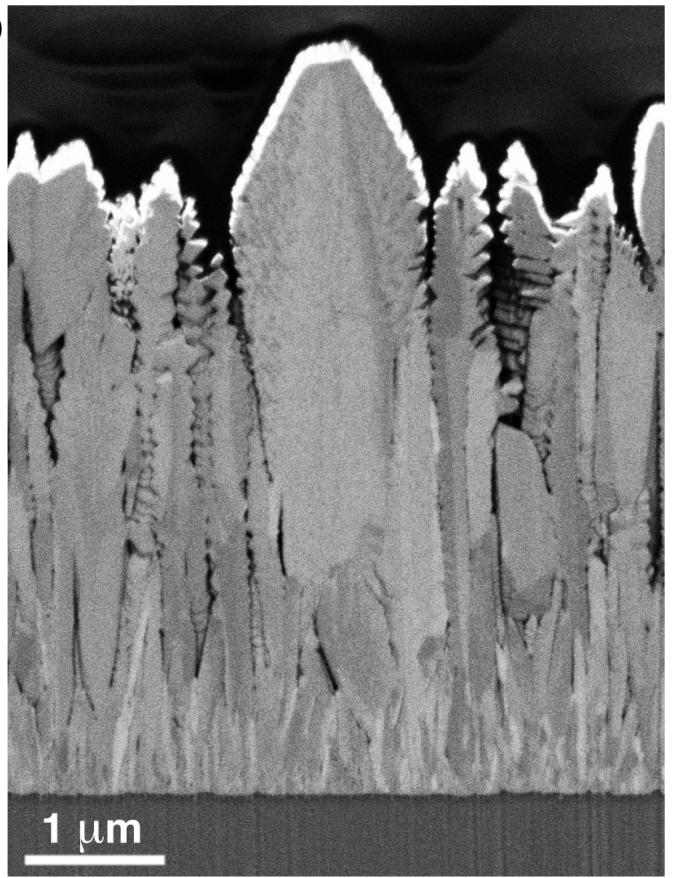

(b)

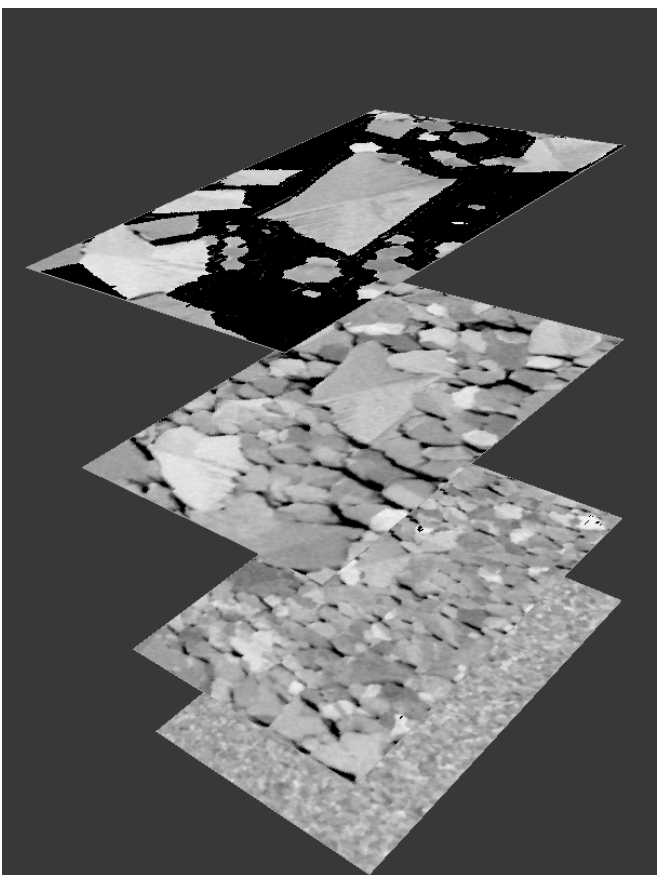

FIG. 2. FIB-SEM cross-section grain structure image of a $\mathrm{ZnO}$ test film (a); compared to typical pyramidal $\mathrm{ZnO}$ for cells, its unusual structure results from very non-standard LP-CVD conditions for growth studies. Lateral slices of tomographic data from the same region (b). Rotational symmetry seen around an axial twin plane in the central pyramid is not visible in one cross-section alone. 